\title{
Aberrant Cutaneous Nerve Loops in the Axilla
}

\author{
Vishwajit Ravindra Deshmukh, Harshita Bhardwaj, Feroz Khan, Tony George Jacob*
}

\begin{abstract}
During routine dissection classes, conducted for first year undergraduate medical students, we encountered a rare anatomical variation in relation to the intercostobrachial nerve (ICBN). The ICBN represents the lateral undivided cutaneous branch of second intercostal nerve. In this case, the ICBN formed nerve loops with branches of the lateral cutaneous branch of the third intercostal nerve. These loops eventually gave branches that probably supplied the floor of the axilla and proximal arm. Nowadays, this ICBN is gaining clinical importance during the axillary lymph node dissections and mammary gland surgeries. Damage to the ICBN, may results in the sensory deficits in patients undergoing surgery. In our case report, ICBN was making aberrant nerve loop along with the branches from the third intercostal nerve. Knowledge regarding the origin, formation and route of ICBN is of clinical significance to axillary surgeons, radiologist and anesthesiologists.
\end{abstract}

\section{KEYWORDS}

axilla; lymph node; nerve plexus; intercostobrachial nerve; surgery

\section{AUTHOR AFFILIATIONS}

Department of Anatomy, All India Institute of Medical Sciences, New Delhi, India

* Corresponding author: Room no. 55, Ground floor, Department of Anatomy, All India Institute of Medical Sciences, New Delhi, India; e-mail: tonygeorgejacob@gmail.com

Received: 5 January 2017

Accepted: 7 April 2017

Published online: 7 June 2017

Acta Medica (Hradec Králové) 2017; 60(1): 51-54

https://doi.org/10.14712/18059694.2017.50

(c) 2017 The Authors. This is an open-access article distributed under the terms of the Creative Commons Attribution License (http://creativecommons.org/licenses/by/4.0), which permits unrestricted use, distribution, and reproduction in any medium, provided the original author and source are credited. 

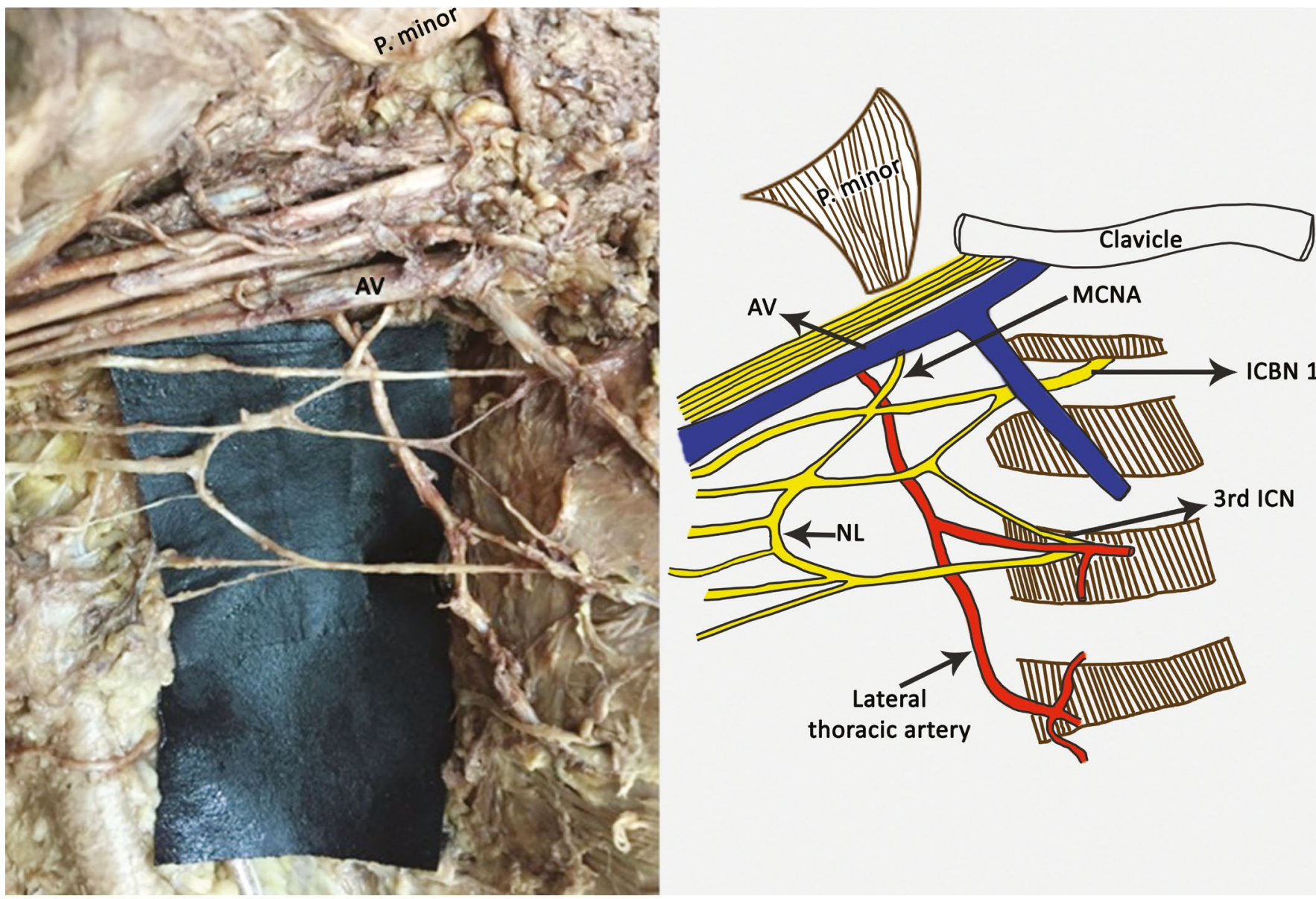

Fig. 1: Photograph showing the formation of nerve loop from the ICBN and third intercostal nerve. Along with nerve loop, branch of lateral thoracic artery is passing between the two-nerve roots of third intercostal nerve (3rd ICN). MCNA: Medial cutaneous nerve of arm; ICBN 1: Intercostobrachial nerve 1; AV: Axillary vein; NL: Nerve loop; P. minor: Pectoralis minor.

\section{INTRODUCTION}

The axilla is a truncated space, forming the passage for neurovascular structures between the upper limb, neck, and thoracic cavity. Variations in the neurovascular structures in this region may affect the outcome of axillary surgeries such as sentinel node identification and biopsy as well as axillary node dissection during a mastectomy for malignancies of the breast (1).

The cords (lateral, medial and posterior) of the brachial plexus in the axilla can be identified with the help of their relative positions to the axillary artery. The three cords enter the axilla at the apex and are arranged, according to the names, around the second and third parts of the axillary artery. In relation to the first part of the artery, however, the lateral and posterior cords are lateral, and the medial cord lies posterior to the artery (2). The lateral cutaneous branch of the second intercostal nerve is termed as the intercostobrachial nerve (ICBN) and can be identified easily because it emerges from the second intercostal space near the mid-axillary line (midway between the anterior and posterior axillary folds) (3). The ICBN is nearly parallel to the axillary vein, at a distance of about 1.5 centimeters (4). The ICBN pierces the intercostal muscles of second intercostal space and serratus anterior muscle around the midaxillary line, crosses the axilla and finally joins with the medial cutaneous nerve of the arm (MCNA). When the nerve passes through the axilla, it gives off the posterior axillary branch, which innervates the skin of the posterior axillary fold. Thereafter, it gives sensory innervations to the skin of upper half of medial and posterior part of the arm (up to the apex of axilla), where it joins with the posterior cutaneous nerve of the arm, which is a branch of the radial nerve (5). Knowledge of the origin of ICBN and its branching pattern is necessary to avoid injury to it during axillary node dissection and mastectomy procedures. There is a paucity of information in published literature regarding the route, location, branching pattern and formation of a plexus or nerve loops in the axilla.

Axillary lymphadenectomy is done for sentinel lymph node identification in mammary gland malignancies or as a component of surgical treatment offered to patients with breast cancers or suspected cancers (6). Post-surgical morbidities in axillary node dissection are restricted mobility of shoulder and arm, sensory changes, pain, and lymphedema. It is reported that in patients with breast cancer, even a sentinel lymph node biopsy can result in the chronic sequelae, such as limitation in arm abduction (0-41.4\%), pain (5.6-51.1\%), paresthesia (5.1-51.1\%) and lymphedema (0-27.3\%) (7). These result from accidental harm to nerves, arteries and lymph vessels, respectively. Preservation of nerves during surgery can significantly decrease alterations of pain sensitivity in the arm after surgery (8). 
Therefore, in this case report, we present a peculiar pattern of formation and distribution of the ICBN that may add to the body of literature and may alert axillary surgeons about possible variations to beware of to reduce the chronic sequelae of nerve injury in their patients.

\section{CASE REPORT}

During routine dissection of the axilla, we encountered the variation of ICBN in right upper limb of a female cadaver, who was 60 years of age at her time of death. The donor had died of natural causes. The cadaver had no visible signs of trauma, surgery or other pathological lesions in the neck, axilla, and thorax. Here, the ICBN after piercing intercostal muscles and serratus anterior muscle, bifurcated into two branches. The larger of the two branches joined with the medial cutaneous nerve of the arm and continued posteriorly towards the axilla, where it probably ended by supplying the skin of the posterior part of the arm. It did not have any branches innervating muscles of the region. The smaller branch of the ICBN joined the lateral cutaneous branch of the third intercostal nerve arising from the 3 rd intercostal space in the mid-axillary line and formed a proximal nerve loop (Figure 1). A distal nerve loop was also formed between the larger and smaller branches of the ICBN and the lateral cutaneous branch of the third intercostal nerve. This distal nerve loop was found in the axilla and this loop gave off five branches that probably innervated the skin of the floor of the axilla and the upper medial part of the arm. None of the dissected branches of the loop or the parent nerves entered any of the muscles surrounding the axilla. The lateral thoracic artery passed deep to the nerve loops in the axilla, but a branch from this artery passed beside the roots of the lateral cutaneous branch of the third intercostal nerve.

\section{DISCUSSION}

Here, we report the formation of aberrant nerve loops and their branches between the lateral cutaneous branches of the second and third intercostal nerves in the axilla. The intercostobrachial nerve is an undivided lateral cutaneous branch of the second intercostal nerve (3). Cunnick grouped the anatomical variation of this nerve into various subcategories: in typel the ICBN arises from T2 and does not give off any branches; type 2 arises from T2 alone and divides into a large main trunk and a much smaller branch; in type 3 the ICBN arises from T2 alone and then divides equally into two branches; in type 4 the ICBN is formed by two equally sized branches from the $\mathrm{T} 1$ and $\mathrm{T} 2$ nerves; in type 5 the nerve arises from two separate T2 radicals to form a single nerve, which does not give off any branches in the axilla and lastly in type 6 it arises from T2 alone and is divided into the large main trunk and at least two smaller branches. The ICBN innervates the skin of the medial and posterior part of the arm and also communicates with the medial cutaneous nerve of the arm (MCNA) (9).

Breast cancer is the most common diagnosed malignancy in women worldwide (22\%) and in India (18.5\%) it ranks second to cervical cancer (10). The ICBN is closely related to axillary lymph nodes, which are traditionally divided into three levels (I, II, III) by pectoralis minor muscle. Nowadays, it is commonly accepted principle that during surgery, lymph node should be extracted from level I to level III (11). Anatomy of the ICBN is highly variable and it divides the axillary space into lower and upper compartments. The upper compartment includes level III and a large part of level II lymph nodes. ICBN is commonly injured during surgeries of the mammary gland and axilla and recent studies have been mainly focused on preservation of ICBN during breast and axillary surgeries (12). According to the observation by $\mathrm{Li} \mathrm{J}$ et al, if the axillary lymph nodes lying above the ICBN have micro or macrometastasis then there were metastasis positive nodes under the ICBN and similarly, if no metastasis is seen in lymph nodes under the ICBN, the upper nodes were also metastasis-free. Thus, ICBN forms the good anatomical landmark to define the axillary lymph node dissection procedure (13). Preservation of ICBN is beneficial to the patient because this leads to a significant decrease in pain sensitivity of the arm, without increasing the duration of the operation or the occurrence of relapses $(14,15)$. Some studies reported that preservation of ICBN consumes much time during mammary gland surgery but in the postoperative period there is the significant decrease in patients sensory deficits and pain (16). In addition, in patients with ICB Neuralgia, anaesthetists have started using ultrasound guided nerve blocks in the subpectoral plance. Aberrant branching patterns would affect the outcome of these procedures that bring relief to the patient (17).

The embryological basis for the formation of aberrant nerve plexus is not fully understood. Various theories have been proposed including the cell signaling. During the fifth week of development, the axons of nerves grow distally to initiate contact with developing limb bud, improper signaling can lead to the formation of aberrant nerve plexus (18). The factors which guide the nerve growth are chemo-attractants (netrins) and repellents (semaphorins and ephrins) which ultimately direct cell processes for appropriate location (19). Another theory includes the improper balance of calcium, which is required for the guidance molecule to work effectively and also to stabilize the microtubules. Microtubule misalignment initiates the development of nerve in the new direction forming the aberrant nerve plexus of the body (20).

This anomaly that we have reported here, has not been mentioned before in literature, even in the studies dedicated to the anatomy of the ICBN. We believe that knowledge of this anomaly may be important to a surgeon during dissection of the axilla and would decrease post-operative morbidity for the patient.

\section{REFERENCES}

1. Rao R, Euhus D, Mayo HG, Balch C. Axillary node interventions in breast cancer: a systematic review. Journal of American Medical Association $2013 ; 310(13)$ : 1385-94.

2. Wildsmith JAW, Armitage EN, McClure JH. Principles and Practice of Regional Anaesthesia. 3rd ed. New York, USA: Churchill Livingstone, 2003.

3. McMinn RM. Upper limb. In: McMinn RM, editor. Last's Anatomy Regional and Applied. 9th ed. Edinburgh: Churchill Livingstone, 1994:82. 
4. Loukas M, Hullett J, Louis RG, Holdman S, Holdman D. The gross anatomy of the extrathoracic course of the intercostobrachial nerve. Clinical Anatomy 2006; 19(2): 106-11.

5. Baker RJ, Fischer JE. Segmental mastectomy and axillary dissection. In: Baker RJ, Fischer JE, eds. Master of Surgery. Philadelphia: Lippincott, William and Wilkins, 2001; 591-3.

6. Soares EW, Nagai HM, Bredt LC, da Cunha AD, Andrade RJ, Soares GV. Morbidity after conventional dissection of axillary lymph nodes in breast cancer patients. World Journal of Surgical Oncology 2014; 12(1): 1 .

7. Verbelen H, Gebruers N, Eeckhout FM, Verlinden K, Tjalma W. Shoulder and arm morbidity in sentinel node-negative breast cancer patients: a systematic review. Breast Cancer Research and Treatment 2014; 144(1): 21-31.

8. Carpenter JS, Sloan P, Andrykowski MA, McGrath P, Sloan D, Rexford T, Kenady D. Risk factors for pain after mastectomy/lumpectomy. Cancer Practice 1999; 7(2): 66-70.

9. Cunnick GH, Upponi S, Wishart GC. Anatomical variants of the intercostobrachial nerve encountered during axillary dissection. The Breast 2001; 10(2): 160-2.

10. Kamath R, Mahajan KS, Ashok L, Sanal TS. A study on risk factors of breast cancer among patients attending the tertiary care hospital, in udupi district. Indian Journal of Community Medicine 2013; 38(2): 95.

11. Masuda N, Tamaki Y, Noguchi S. Management of axillary and internal mammary lymph nodes in primary breast cancer. Nihon Geka Gakkai Zasshi 2001; 102(6): 465-72.

12. Pain SJ, Vowler S, Purushotham AD. Axillary vein abnormalities contribute to development of lymphoedema after surgery for breast cancer. British Journal of Surgery 2005; $92(3)$ : 311-5.
13. Li J, Zhang Y, Zhang W, Jia S, Gu X, Ma Y, Li D. Intercostobrachial nerves as a novel anatomic landmark for dividing the axillary space in lymph node dissection. ISRN Oncology 2013; 2013.

14. Torresan RZ, Cabello C, Conde DM, Brenelli HB. Impact of the preservation of the intercostobrachial nerve in axillary lymphadenectomy due to breast cancer. The Breast Journal 2003; 9(5): 389-92.

15. Seidel R, Gray AT, Wree A, Schulze M. Surgery of the axilla with combined brachial plexus and intercostobrachial nerve block in subpectoral intercostal plane. British Journal of Anaesthesia 2017; 118(3):472-4.

16. Freeman SR, Washington SJ, Pritchard T, Barr L, Baildam AD, Bundred NJ. Long term results of a randomised prospective study of preservation of the intercostobrachial nerve. European Journal of Surgical Oncology 2003; 29(3): 213-5.

17. Wisotzky EM, Saini V, Kao C. Ultrasound-guided intercostobrachial nerve block for intercostobrachial neuralgia in breast cancer patients: a case series. Physical Medicine and Rehabilitation 2016; $8(3): 273-7$

18. Tatar I, Brohi R, Sen F, Tonak A, Celik H. Innervation of the coracobrachialis muscle by a branch from the lateral root of the median nerve. Folia Morphol (Warsz) 2004; 63(4): 503-6.

19. Budhiraja V, Rastogi R, Asthana AK, Sinha P, Krishna A, Trivedi V. Concurrent variations of median and musculocutaneous nerves and their clinical correlation-a cadaveric study. Italian Journal of Anatomy and Embryology 2011; 116(2): 67.

20. Schoenwolf GC, Bleyl SB, Brauer PR, Francis-west PH. Development of the peripheral nervous system. In: Schoenwolf GC, Editor. Larsen's Human Embryology. Philadelphia. 